\title{
Prognostic model for long-term survival of locally advanced non-small-cell lung cancer patients after neoadjuvant radiochemotherapy and resection integrating clinical and histopathologic factors
}

Christoph Pöttgen ${ }^{1}$, Martin Stuschke ${ }^{1,8^{*}}$, Britta Graupner ${ }^{1}$, Dirk Theegarten² ${ }^{2}$ Thomas Gauler $^{3}$, Verena Jendrossek ${ }^{4}$, Lutz Freitag ${ }^{5}$, Jehad Abu Jawad', Eleni Gkika', Jeremias Wohlschlaeger², Stefan Welter ${ }^{6}$, Matthias Hoiczyk ${ }^{3}$, Martin Schuler ${ }^{3,7,8}$, Georgios Stamatis ${ }^{6}$ and Wilfried Eberhardt ${ }^{3,6}$

\begin{abstract}
Background: Outcome of consecutive patients with locally advanced non-small cell lung cancer and histopathologically proven mediastional lymph node metastases treated with induction chemotherapy, neoadjuvant radiochemotherapy and thoracotomy at the West German Cancer Center between 08/2000 and 06/2012 was analysed. A clinicopathological prognostic model for survival was built including partial or complete response according to computed tomography imaging $(C T)$ as clinical parameters as well as pathologic complete remission (pCR) and mediastinal nodal clearance (MNC) as histopathologic factors.
\end{abstract}

Methods: Proportional hazard analysis (PHA) and recursive partitioning analysis (RPA) were used to identify prognostic factors for survival. Long-term survival was defined as survival $\geq 36$ months.

Results: A total of 157 patients were treated, median follow-up was 97 months. Among these patients, pCR and MNC were observed in 41 and 85 patients, respectively. Overall survival was $56 \pm 4 \%$ and $36 \pm 4 \%$ at 24 and 60 months, respectively. Sensitivities of $\mathrm{PCR}$ and MNC to detect long-term survivors were $38 \%$ and $61 \%$, specificities were $84 \%$ and $52 \%$, respectively.

Multivariable survival analysis revealed pCR, cN3 category, and gender, as prognostic factors at a level of $a<0.05$. Considering only preoperative available parameters, CT response became significant. Classifying patients with a predicted hazard above the median as high risk group and the remaining as low risk patients yielded better separation of the survival curves by the inclusion of histopathologic factors than by preoperative factors alone ( $p<0.0001$, log rank test). Using RPA, pCR was identified as the top prognostic factor above clinical factors $(p=0.0006)$. No long term survivors were observed in patients with cT3-4 CN3 tumors without $p C R$.

Conclusions: $p C R$ is the dominant histopathologic response parameter and improves prognostic classifiers, based on clinical parameters. The validated prognostic model can be used to estimate individual prognosis and forms a basis for patient selection for treatment intensification.

Keywords: Prognostic model, Long-term survival, NSCLC, Histopathological response

\footnotetext{
* Correspondence: martin.stuschke@uk-essen.de

'Department of Radiotherapy, West German Cancer Center, University of Duisburg-Essen, Essen, Germany

${ }^{8}$ German Cancer Consortium (DKTK), Heidelberg, Germany

Full list of author information is available at the end of the article
} 


\section{Background}

Mediastinal nodal clearance (MNC) defined as sterilization of initially involved (cN2, cN3) mediastinal lymph nodes after neoadjuvant radiochemotherapy followed by surgery (ypN0) was identified as a prognostic marker for long-term survival for stage III non-small-cell lung cancer (NSCLC) [1-5]. In these trials, the proportion of patients with MNC among patients who received thoracotomies after neoadjuvant radiochemotherapy was approximately $50 \%$. MNC was taken as a surrogate endpoint for the treatment effect of neoadjuvant radiochemotherapy and represented the primary endpoint of the Radiation Therapy Oncology Group phase II trial 02-29 [6]. In this trial, full dose radiotherapy to $61.2 \mathrm{~Gy}$ in conventional fractionation and weekly concurrent carboplatin/paclitaxel chemotherapy was given. Nodal clearance was achieved in $63 \%$ of patients with pathologically proven $\mathrm{N} 2$ or N3 involvement receiving thoracotomy, but only $8 \%$ had a pathologic complete remission (pCR), defined as sterilization of tumor tissue at all involved sites (primary and lymph nodes). Generally, pCR is found at lower frequencies than $\mathrm{MNC}$ in patients undergoing thoracotomy after neoadjuvant radiochemotherapy and has been observed in 8-39\% of patients in larger series $[1,2,5-10]$. Others found that $\mathrm{pCR}$ is an important prognostic factor for survival after neoadjuvant radiochemotherapy and resection [9-11].

In this large retrospective monoinstitutional study, we evaluated the prognostic strengths of patient characteristics, treatment variables and histopathologic response parameters within a prognostic model for survival in a comprehensive group of patients with locally advanced NSCLC and histopathologically proven mediastinal lymph node metastases undergoing neoadjuvant radiochemotherapy and thoracotomy. In particular, the prognostic values of $\mathrm{pCR}$ and $\mathrm{MNC}$ on long-term survival were analyzed.

\section{Methods}

This observational study included data from consecutive patients with stage IIIA/IIIB NSCLC according to the UICC classification, 7th edition 2009, who were treated at the West German Cancer Center between August 2000 and June 2012.

The study has been approved by the Ethics committee of the University of Duisburg-Essen, Essen, Germany. All treatments were performed in accordance with the German Legislation of Radiation Protection. Written informed consent was obtained from all patients prior to treatment initiation.

Following induction chemotherapy and neoadjuvant chemoradiotherapy, all patients were resected at the Division of Thoracic Surgery, Ruhrlandklinik, Essen. All patients were staged by mediastinoscopy or endobronchial ultrasound-guided needle aspiration to confirm $\mathrm{cN} 2$ or
cN3 status by histo- or cytopathology. The additional workup included computed tomography (CT) scans of the thorax and abdomen and bone scans, or whole body $\mathrm{PET} / \mathrm{CT}$ which became available at our institution in 2002. In addition, CT or MRI scans of the brain were performed in all patients. All patients were discussed in the multidisciplinary tumor board of the lung cancer center of the West German Cancer Center. Following three cycles of induction chemotherapy (cisplatin/paclitaxel doublets predominantly given throughout the whole time period, cisplatin/etoposide was administered to patients prior to 2007) concurrent radiochemotherapy was initiated [7]. Cisplatinum-based doublets were used during the concurrent chemoradiotherapy phase. Before 2004, etoposide was predominantly applied which was later replaced by vinorelbine as combination partner for cisplatin. Patients received conventionally fractionated radiotherapy to $44-46$ Gy at 2 Gy per fraction, or accelerated hyperfractionated radiotherapy at $2 \times 1.5$ Gy per day, $\geq 6 \mathrm{~h}$ interval, 5 days a week, up to a total dose of 45 Gy. Threedimensional treatment planning was performed for all patients based on planning CT scans following induction chemotherapy. The planning procedure has been previously described in detail [7].

During the last week of neoadjuvant radiochemotherapy, patients underwent restaging CT-scans and were again discussed in the multidisciplinary tumor board. Preoperatively, re-mediastinoscopy was performed in all patients with initial N3-disease to define the response to induction treatment. Patients with positive contralateral nodes at repeated mediastinoscopy were excluded from surgery and were offered definitive concurrent radiochemotherapy. Those patients who were evaluated as resectable by the thoracic surgeon at that time point were offered surgery. Criteria of resectability have been described earlier [12]. Patients underwent a preoperative cardiovascular risk assessment including a cardiopulmonary function test. Patients were ineligible due to a predicted postoperative forced expiratory volume at $1 \mathrm{~s}$ of less than 1 liter (quantitative ventilation-perfusion lung scanning), cardiac infarction or unstable angina pectoris during the 6 months before study entry or cardiac failure of class III or greater (NYHA criteria). If re-evaluation showed continuing medical/functional and technical operability, patients were taken to thoracotomy 3-5 weeks after the end of radiation treatment.

After treatment completion, no adjuvant treatment was planned and patients were monitored every three months during the first year of follow-up. Thereafter, examination intervals were set to 4-6 months. Time to progression (TTP) and overall survival (OS) were calculated from the start of the induction chemotherapy. For non-progressing patients, TTP was calculated as censored at the time of the last follow-up visit. Survival 
information was updated from the local residents registration offices between 1st and $15^{\text {th }}$ of September 2013. Toxicities were assessed using CTC (v. 2) scores [13].

Pathologic complete response ( $\mathrm{pCR}$ ) was defined as complete disappearance of vital tumor cells at all initially involved tumor sites (ypT0 ypN0) assessed by histopathologic examination of the resection specimen. Regression grades $2 \mathrm{~A}$ and $2 \mathrm{~B}$ were defined as evidence of therapy-induced tumor regression with $>10 \%$ and $<10 \%$ of vital tumor cells remaining, respectively [14]. MNC was defined as ypNo. Histopathologic complete regression at the primary tumor site only (pCR-T) was defined as ypT0.

Long-term survivors were defined as patients living $\geq 36$ months since start of induction chemotherapy. Twenty percent of all deceased patients $(n=98)$ of this cohort fulfilled the criteria for long-term survivors. On the other hand, $47 \%$ of the living patients had a followup time of less than 36 months. For these patients, the conditional probability p was calculated to survive 3 years having the observed survival time according to a backward selection proportional hazard model. These patients were included in the analysis with weight $\mathrm{p}$ as long-term survivors and with weight 1-p as short-term survivors, respectively.

Sensitivities and specificities of the histopathologic regression parameters to predict long-term survival as well as the positive likelihood ratio = sensitivity/(1-specificity) together with its 95\% confidence limits and the CochraneMantel-Haenszel $(\mathrm{CMH})$ test for the association of the histopathologic response parameters with survival were calculated with the procedure FREQ using SAS statistical software version 9.2 (Cary, NC). Proportional hazard regression analysis (PHA) was performed with the procedure PHREG. The following clinical explanatory variables were included in the full proportional hazard regression model. Patient-dependent variables: age as a continuous variable, gender, Charlson comorbidity score. Tumor characteristics: cN2 (yes vs. no), cN3, Pancoast tumor localisation, cT3, cT4, stage IIB, adenocarcinoma (yes vs. no), squamous cell carcinoma (yes vs. no), grade 3 carcinoma, number of histopathologically proven mediastinal lymph node metastases at initial staging. Treatment characteristics: hyperfractionated accelerated radiotherapy (yes vs. no), cisplatin/paclitaxel doublet as induction chemotherapy, cisplatin/vinorelbine as concurrent chemotherapy, pneumonectomy, R1 resection, R2 resection. In addition, the following histopathologic and clinical response variables were included: $\mathrm{pCR}, \mathrm{MNC}$, regression grade (regression grade $\geq 2 \mathrm{~B}$, regression grade $>2 \mathrm{~A}, \mathrm{ypT}$ ), response to neoadjuvant radiochemotherapy according to computed tomography imaging studies before and after neoadjuvant radiochemotherapy according to the RECIST criteria (PD/ $\mathrm{NC}$ versus $\mathrm{PR} / \mathrm{CR}$ ) [15]. A backward variable elimination procedure was used to retain variables in the model significant at $\alpha=0.05$. The proportional hazard assumption was studied by Schoenfeld partial residuals and their correlation with the rank order of failure times and by introducing time dependent interaction terms for the covariates in the model to detect a possible trend over time of the hazard ratio $[16,17]$.

Prognostic models were derived using the PHA variable estimates after backward elimination with or without inclusion of the histopathologic parameters. The sample was split in equally sized high risk or low risk groups depending on whether the prognostic index, defined as the vector product of the PHA regression coefficients and the patients' expression of prognostic parameters constituting a factor of the hazard function, was located above or below the median, respectively. Ten-fold cross-validation was used to evaluate the predictive accuracy of the prognostic model on a data set independent of the one used for model building. Therefore, the whole sample of $157 \mathrm{pa}$ tients was split into 10 approximately equally sized subgroups by random numbers. For each $k$-th subgroup, the prognostic model was developed from scratch on the basis of the data from the 9 other subgroups by fitting the PHA model with backward elimination. This model was then used to split the k-th subgroup into a high risk and low risk group. Not necessarily the same variables had to stay in the model for each of the $\mathrm{k}$ loops of cross-validation. Repeating this process for all subgroups resulted in a split of the whole sample into a high and a low risk group. The respective Kaplan-Meier curves were termed as crossvalidated Kaplan Meier curves [18]. The prognostic model containing clinical and histopathological covariables was evaluated against the standard model using the difference of the log-rank statistics for comparison of the crossvalidated Kaplan Meier curves of the high and low risk groups according to both models as a test statistic [18].

For regression tree growing during recursive partitioning analysis (RPA), the log rank test was used as the splitting criterion [19]. The dichotomic prognostic parameter that results in the largest separation of 5 year survival at each node with a p-value $<0.05$ was selected as the criterion for this node.

\section{Results}

Median follow-up for survival of the entire cohort was 97 months. Patient characteristics have been reported in a previous publication on the effects of accelerated hyperfractionation on $\mathrm{pCR}$ and are summarized in Table 1 [7]. One hundred fifty-seven patients have been resected out of 164 who underwent the induction chemoradiotherapy phase completely. Eight patients were not resected either due to comorbidities $(n=3)$, persistent contralateral lymph node involvement at remediastinoscopy $(\mathrm{n}=3)$, or patients' refusal $(\mathrm{n}=2)$, and received 
Table 1 Patient characteristics

\begin{tabular}{|c|c|c|}
\hline Total & $\begin{array}{l}\text { No. } \\
157\end{array}$ & $\begin{array}{c}{[\%]} \\
100 \\
\end{array}$ \\
\hline \multicolumn{3}{|l|}{ Sex } \\
\hline Male/Female & $94 / 63$ & $60 / 40$ \\
\hline \multicolumn{3}{|l|}{ Age, years } \\
\hline median (range) & \multicolumn{2}{|c|}{$59 \cdot 0(34-74)$} \\
\hline \multicolumn{3}{|c|}{ Primary tumor and lymph node classification } \\
\hline cT1-3 N2 & 81 & 52 \\
\hline cT1-3 N3 & 35 & 22 \\
\hline CT4 N2 & 37 & 24 \\
\hline cT4 N3 & 4 & 2 \\
\hline \multicolumn{3}{|l|}{ Histology } \\
\hline Squamous Cell Carcinoma & 58 & 37 \\
\hline Adenocarcinoma & 75 & 48 \\
\hline Large Cell Carcinoma & 16 & 10 \\
\hline not differentiated, NOS & 7 & 4 \\
\hline sarcomatoid & 1 & 1 \\
\hline \multicolumn{3}{|l|}{ G } \\
\hline 1 & 3 & 2 \\
\hline 2 & 50 & 32 \\
\hline 3 & 94 & 60 \\
\hline$x$ & 10 & 6 \\
\hline \multicolumn{3}{|l|}{ Localisation } \\
\hline Sulcus Superior (Pancoast) & 9 & 6 \\
\hline Upper Lobe & 95 & 60 \\
\hline Middle Lobe/Centrally located & 30 & 19 \\
\hline Lower Lobe & 23 & 15 \\
\hline \multicolumn{3}{|l|}{ Induction Chemotherapy } \\
\hline Cisplatin/Etoposide & 11 & 7 \\
\hline Cisplatin/Paclitaxel & 146 & 93 \\
\hline \multicolumn{3}{|l|}{ Concurrent Chemotherapy } \\
\hline Cisplatin/Etoposide & 28 & 18 \\
\hline Cisplatin/Vinorelbine & 129 & 82 \\
\hline \multicolumn{3}{|l|}{ Radiotherapy Fractionation } \\
\hline conventional & 77 & 49 \\
\hline accelerated-hyperfractionated & 80 & 51 \\
\hline \multicolumn{3}{|l|}{ Surgery } \\
\hline Segmentectomy & 2 & 1 \\
\hline Lobectomy & 105 & 68 \\
\hline Bi-Lobectomy & 7 & 4 \\
\hline Pneumonectomy & 40 & 26 \\
\hline Thoracotomy without resection & 1 & 1 \\
\hline
\end{tabular}

Table 1 Patient characteristics (Continued)

\begin{tabular}{lll}
\hline Resection & & \\
microscopic complete (R0) & 143 & 92 \\
microscopic incomplete (R1) & 9 & 6 \\
macroscopic incomplete (R2) & 4 & 2 \\
\hline
\end{tabular}

definitive radiochemotherapy $(n=5)$, or palliative treatment $(n=3)$, respectively. Pneumonectomies were performed in 40 patients. In this cohort, none of the patients died within 30 days after surgery. Figure 1 shows the survival curve of all patients who underwent tumor resection ( $\mathrm{n}=157)$. Two-, three-, and five- year survival was $56 \pm$ $4 \%, 46 \pm 4 \%$, and $36 \pm 4 \%$, respectively.

\section{Sensitivity and specificity of histopathologic regression parameters to predict long-term survival}

Sensitivity and specificity as well as the likelihood ratio were analyzed for the different histopathologic regression parameters to predict long-term survival. For the entire group of patients, pCR had the highest likelihood ratio among all histopathologic parameters that were significantly different from the value 1 for a useless test (Table 2). $\mathrm{pCR}$ and pCR-T (pathologic complete remission in the primary tumor in contrast to complete remission of tumor and mediastinal nodes) carry similar information and the vast majority $(n=41)$ of the 46 patients with pCR-T also achieved pCR. Decreasing the cut-off level for histopathologic tumor regression required for a positive histopathologic response from $\mathrm{pCR}$ over regression grades $2 \mathrm{~B}$ to $2 \mathrm{~A}$ (Reg-grade $\geq 2 \mathrm{~B}$ to Reg-grade $\geq 2 \mathrm{~A}$ ) decreased the likelihood ratio for the association of the surrogate marker with long-term survival. Sensitivity of $\mathrm{pCR}$ as a predictor of survival was low (0.38), indicating that the majority of long-term survivors not achieving $\mathrm{pCR}$ following preoperative therapy were salvaged by tumor resection. MNC was found in approximately twice as many patients as pCR-T or pCR. This suggests a higher radiosensitivity of lymph node metastases as compared to the primary tumors. Sensitivity of MNC to predict survival was higher than that of pCR-T or pCR, but specificity was poor. The positive likelihood ratio for MNC was not significantly different from 1 (Table 2).

\section{Proportional hazard analysis of the clinical and pathological variables on survival}

Proportional hazard regression containing all clinical and pathological variables found $\mathrm{pCR}, \mathrm{cN} 3$, and gender as independent prognostic factors for survival using backward elimination of non-significant variables for reduced model selection at a significance level of $\alpha=0.05$ (model (1), Table 3). 


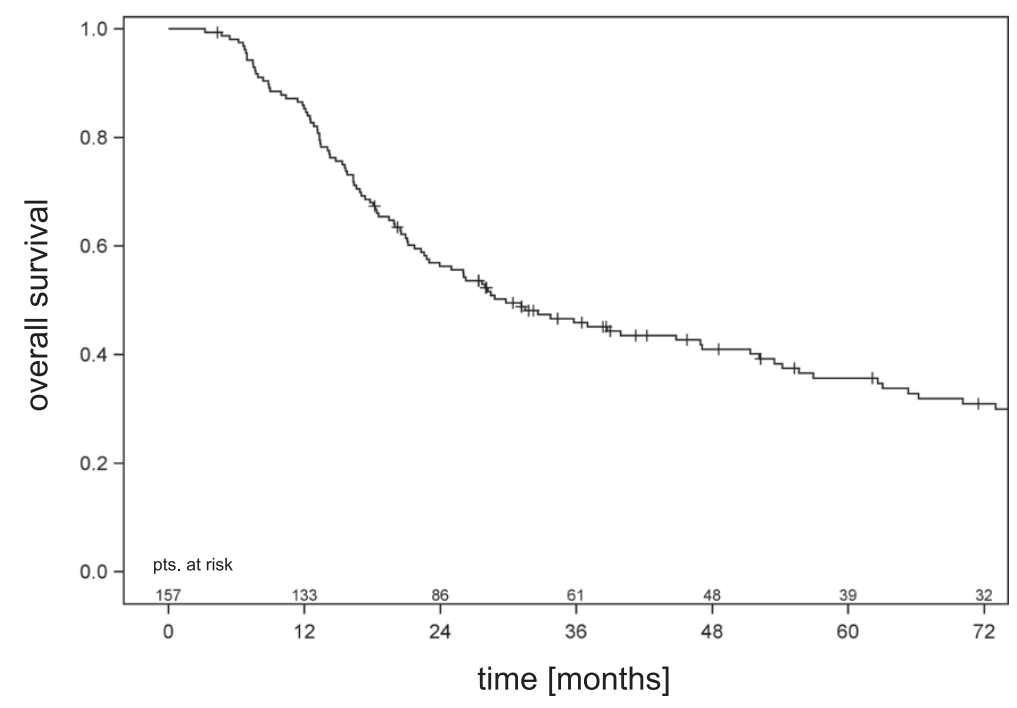

Figure 1 Survival curve for all patients of this study.

The time era of treatment was not significantly associated with survival, indicating the absence of unrecognized period-dependent confounders.

With respect to the other histopathologic parameters, neither MNC without $\mathrm{pCR}$ nor Reg-grade $\geq 2 \mathrm{~B}$, or Reggrade $\geq 2 \mathrm{~A}$ without $\mathrm{pCR}$ carried important prognostic information in addition to pCR. After adjustment of the other prognostic factors, patients with MNC without pCR had a similar prognosis as patients without MNC and without pCR. 15 of 44 patients with MNC but without $\mathrm{pCR}$ were long-term survivors, 16 had known progression of disease (11 at distant and 2 at locoregional sites only, and 3 at both sites).

Response according to CT studies was not selected as an independent prognostic factor in addition to pCR.

No significant deviation from the proportional hazard assumption was found by analysis of the correlation between the Schoenfeld residuals and the rank order of failure times. Furthermore, time dependence of the hazard ratio did not become significant $(\mathrm{p}>0.05)$.
In addition, the PHA parameter estimates from a standard model (model (2)) containing only pretreatment patient and tumor dependent clinical parameters with backward selection are shown in Table 3. Response according to $\mathrm{CT}$ studies became significant in the absence of histopathologic response parameters.

Both models were used to classify patients into equally sized high and low risk groups. A patient was assigned to the high risk group if the estimated hazard according to the model was larger than the median value in the sample of patients. To create continuous predictors, age was added as a continuous variable to the models. Older age was non-significantly associated with poorer survival by a hazard ratio of 1.02 per year increase. The model including histopathologic parameters led to a better separation of the survival curves between the high risk and low risk groups. The log-rank chi-square test statistic for the separation of the survival curves of the high and low risks groups was significantly larger using the model including $\mathrm{pCR}$ than the model including preoperative

Table 2 Sensitivity and specificity for the respective histopathologic response criterium to predict long term survival

\begin{tabular}{|c|c|c|c|c|c|c|}
\hline \multicolumn{7}{|c|}{ Patients with cN2-3 status } \\
\hline & Number of patients & Long-term survivors & Sensitivity & Specificity & Sensitivity/(1-specificity) & CMH-test \\
\hline all patients & 157 & 72 & & & & \\
\hline $\mathrm{pCR}$ & 41 & 28 & $0.38(0.27-0.50)$ & $0.84(0.77-0.92)$ & $2.46(1.39-4.38)$ & $p=0.0012$ \\
\hline Reg-grade $\geq 2 \mathrm{~B}$ & 72 & 40 & $0.56(0.44-0.67)$ & $0.62(0.52-0.73)$ & $1.48(1.05-2.08)$ & $p=0.025$ \\
\hline Reg-grade $\geq 2 \mathrm{~A}$ & 135 & 66 & $0.92(0.85-0.98)$ & $0.19(0.11-0.27)$ & $1.13(0.99-1.28)$ & $p=0.057$ \\
\hline pCR-T & 46 & 29 & $0.40(0.29-0.51)$ & $0.80(0.71-0.88)$ & $1.96(1.18-3.25)$ & $p=0.008$ \\
\hline MNC & 85 & 44 & $0.61(0.50-0.72)$ & $0.52(0.41-0.62)$ & $1.26(0.95-1.69)$ & $p=0.11$ \\
\hline
\end{tabular}

$\mathrm{CMH}$ measures the strength of association between the histopathologic response variable and long term survival which was defined as patient survival $\geq 36$ months. 
Table 3 Significant prognostic variables from proportional hazard regression analysis of survival data

\begin{tabular}{|c|c|c|c|c|}
\hline \multirow[b]{2}{*}{ variable } & \multicolumn{2}{|c|}{ model (1) containing clinical + pathol. covariates } & \multicolumn{2}{|c|}{ standard model (2) containing clinical covariates alone } \\
\hline & Hazard ratio & p-value & Hazard ratio & p-value \\
\hline $\mathrm{pCR}$ & $0.41(0.25-0.67)$ & $p=0.0003$ & not included & \\
\hline $\mathrm{cN3}$ & $1.52(1.01-2.29)$ & $p=0.047$ & $1.59(1.06-2.39)$ & $p=0.027$ \\
\hline gender (female) & $0.57(0.38-0.87)$ & $p=0.008$ & $0.63(0.42-0.94)$ & $p=0.025$ \\
\hline$C T$ - tumor response & Backward eliminated & n.s. & $0.59(0.39-0.91)$ & $p=0.017$ \\
\hline
\end{tabular}

Model (1) contains all clinical and pathological covariates, as described under Methods. Backward elimination of non-significant variables was used for reduced model selection. CT-tumor response: partial or complete response after neoadjuvant radiochemotherapy in comparison to the pretreatment computed tomography imaging study.

available parameters alone ( $\mathrm{p}<0.0001, \mathrm{chi}^{2}$ test). Ten-fold cross-validation was performed in order to estimate the predictive value of both models on a data set independent of that one used for the model building process. The cross-validated Kaplan Meier curves for the high and low risk groups according to the model including histopathologic variables are shown in Figure 2. All 41 with pCR were sorted in the cross validated low risk group. A comparison of the log-rank statistics for the differences between the cross-validated Kaplan Meier curves for the high and low risk groups revealed a better separation by the model including histopathologic parameters than by the model including preoperative available parameters alone ( $\mathrm{p}=0.017, \mathrm{chi}^{2}$ test).

In addition, we analyzed whether tumor response according to the CT-studies after neoadjuvant radiochemotherapy might predict $\mathrm{pCR}$. The Spearman rank correlation between the response according to the CTstudies $(\mathrm{PD} / \mathrm{NC}$ vs. $\mathrm{PR} / \mathrm{CR})$ and $\mathrm{pCR}$ was $0.21(\mathrm{p}<0.05)$. None of the patients in this study, all of whom underwent surgery, had progressive disease after neoadjuvant radiochemotherapy. Among the 123 responding patients, there were 38 with pCR, while among the 34 non-responding patients only 3 were detected with pCR. The prognostic model (2) based on patient and tumor dependent pretreatment factors alone could also be improved by introduction of CT-response as an independent prognostic factor when histopathologic response parameters were not considered for prediction. The backward elimination procedure retained CT-response as a prognostic factor (hazard ratio $0.59(0.39-0.91)$ for responding patients, $\mathrm{p}=0.017$, chi $^{2}$ test $)$.

$\mathrm{PET} / \mathrm{CT}$ investigations before and after induction treatment were only available in less than half of the patients $(n=58)$. In an exploratory subgroup-analysis, deltaSUV $_{\text {max }}$ in the primary tumor $\left(=\left(\mathrm{SUV}_{\max }\right.\right.$ after induction chemotherapy)/(SUV $\mathrm{Sax}_{\max }$ before induction chemotherapy)) showed a positive correlation with $\mathrm{pCR}$ when using a cutoff-level of 0.3 separating responders (deltaSUV $V_{\max }<0.3$ ) from non-responders (deltaSUV $V_{\max } \geq 0.3$;

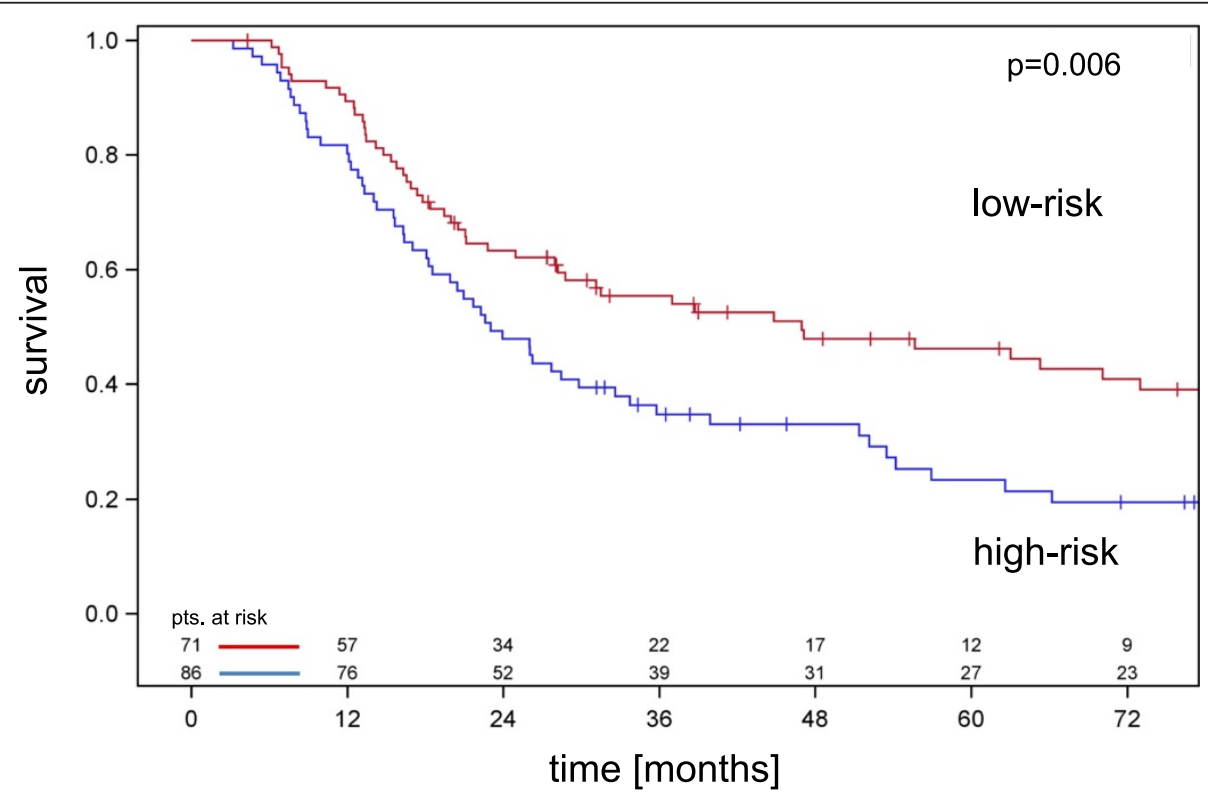

Figure 2 Cross-validated survival curves for patients classified as high and low risk including significant clinical and histopathologic parameters as well as age. The survival difference was significant using the log rank test $(p=0.006)$. 
Spearman rank correlation $0.28, \mathrm{p}=0.033$ ). While 8 patients showed $\mathrm{pCR}$ in 17 responders, only 8 of 41 non-responders were found with pCR. When using CTresponse criteria in this subgroup, $16 \mathrm{pCR}$ patients out of 48 responders versus one $\mathrm{PCR}$ patient out of 10 nonresponders were identified (Spearman rank correlation $0.19, \mathrm{p}=0.097$ ). Due to the small number of patients, deltaSUV $_{\max }$ was not included in the prognostic model for survival.

Recursive partitioning analysis (RPA) including all clinical and pathological predictor variables led to the regression tree shown in Figure 3. The top node contains $\mathrm{pCR}$ as the predictor variable. For patients without pCR, the $\mathrm{cN} 3$ lymph node category became important. For $\mathrm{cN} 3$ patients, high $\mathrm{T}$ category was selected as additional split to obtain prognostic subgroups.

Fourteen long term survivors were among the patients with $\mathrm{cN} 3$ disease. Five of them had a pCR. From the remaining 9 patients, all patients had cT2 tumors and seven showed a PR/CR to induction chemotherapy.

Kaplan Meier survival curves are given in Figure 4 for all patients with $\mathrm{pCR}$ and $\mathrm{cN} 2, \mathrm{pCR}$ and $\mathrm{cN} 3$, not-pCR and $\mathrm{cN} 2$ as well as not-pCR and $\mathrm{cN} 3$ following the splits according to the upper nodes of the regression tree in Figure 3. There was a significant effect of pCR on survival over the strata $\mathrm{cN} 2$ and $\mathrm{cN} 3(\mathrm{p}=0.0004, \log$ rank test) and survival differed between $\mathrm{cN} 2$ and $\mathrm{cN} 3$ patients
( $\mathrm{p}=0.017, \log$ rank test). Survival at 5 years was $61 \pm 4 \%$ for patients with $\mathrm{pCR}$ and $\mathrm{cN} 2,63 \pm 17 \%$ for $\mathrm{pCR}$ and $\mathrm{cN} 3$, and $33 \pm 6 \%$ for not-pCR and cN2, as well as $11 \pm$ $6 \%$ for not-pCR and cN3 patients, respectively.

\section{Proportional hazard analysis of the prognostic value of clinical and pathological characteristics on time to progression}

Proportional hazard analysis revealed that complete remission by RECIST, and pCR were the sole prognostic parameters according to the proportional hazard model using backward selection that predicted time to progression (TTP). The hazard ratio associated with $\mathrm{pCR}$ on TTP was 0.34 (0.16-0.70).

\section{Discussion and conclusion}

Important pre-treatment patient- and tumor-dependent prognostic factors found in this study were the clinical lymph node status, pneumonectomy, gender, adenocarcinoma histology, age, and Pancoast tumor localisation. All patients received induction chemotherapy, neoadjuvant radiochemotherapy und thoracotomy. These findings are in accordance with the Intergroup 0139 trial which also identified female sex as a favorable prognostic factor [2].

pCR was a strong prognostic factor for survival in this analysis and in other studies after neoadjuvant radiochemotherapy for locally advanced non-small cell lung cancer

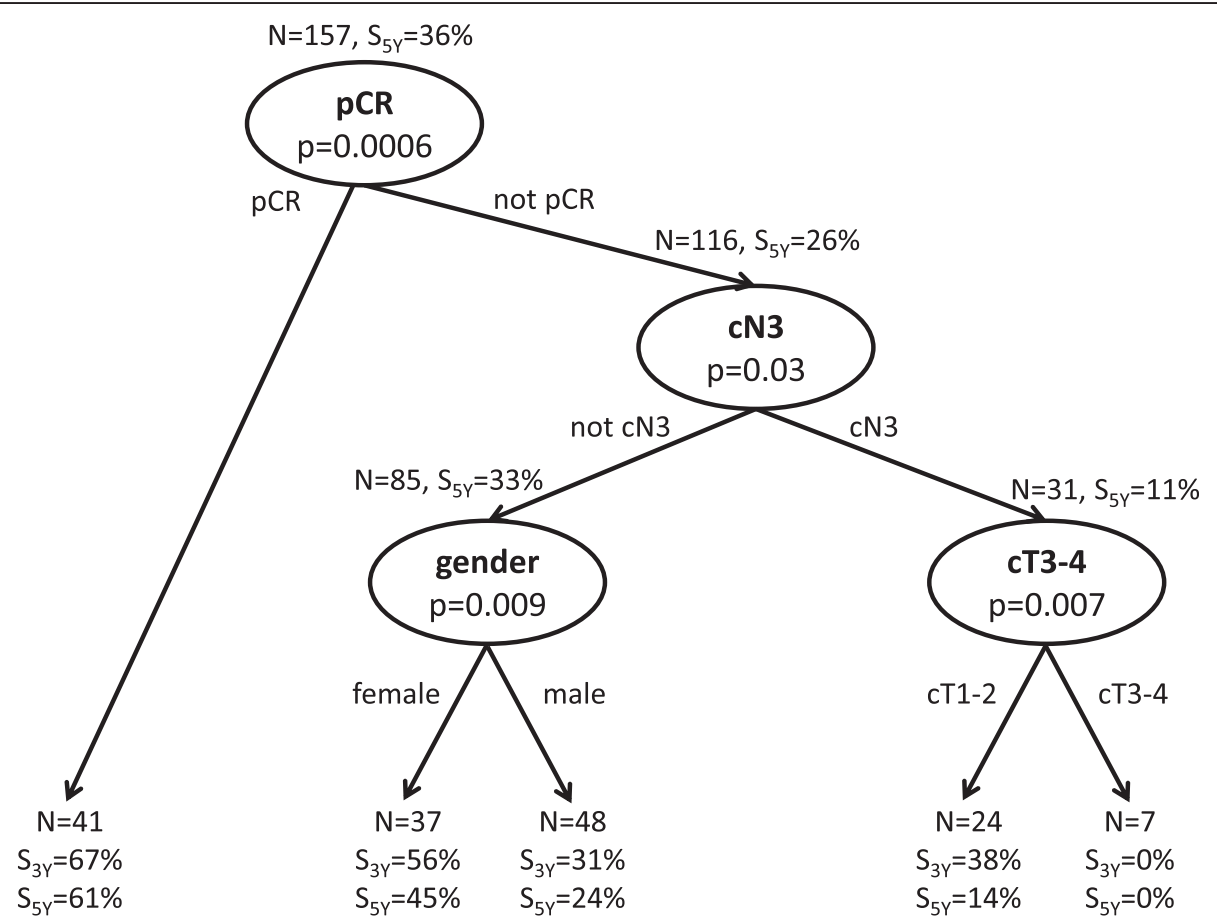

Figure 3 Regression tree diagram from recursive partition analysis using clinical and histopathologic prognostic parameters. For each node, the number of patients for the split, the log rank statistic for dissimilarity between the subgroups, and survival at 3 and 5 years $\left(S_{3 y,} S_{5 y}\right)$ for the subgroups are given. 


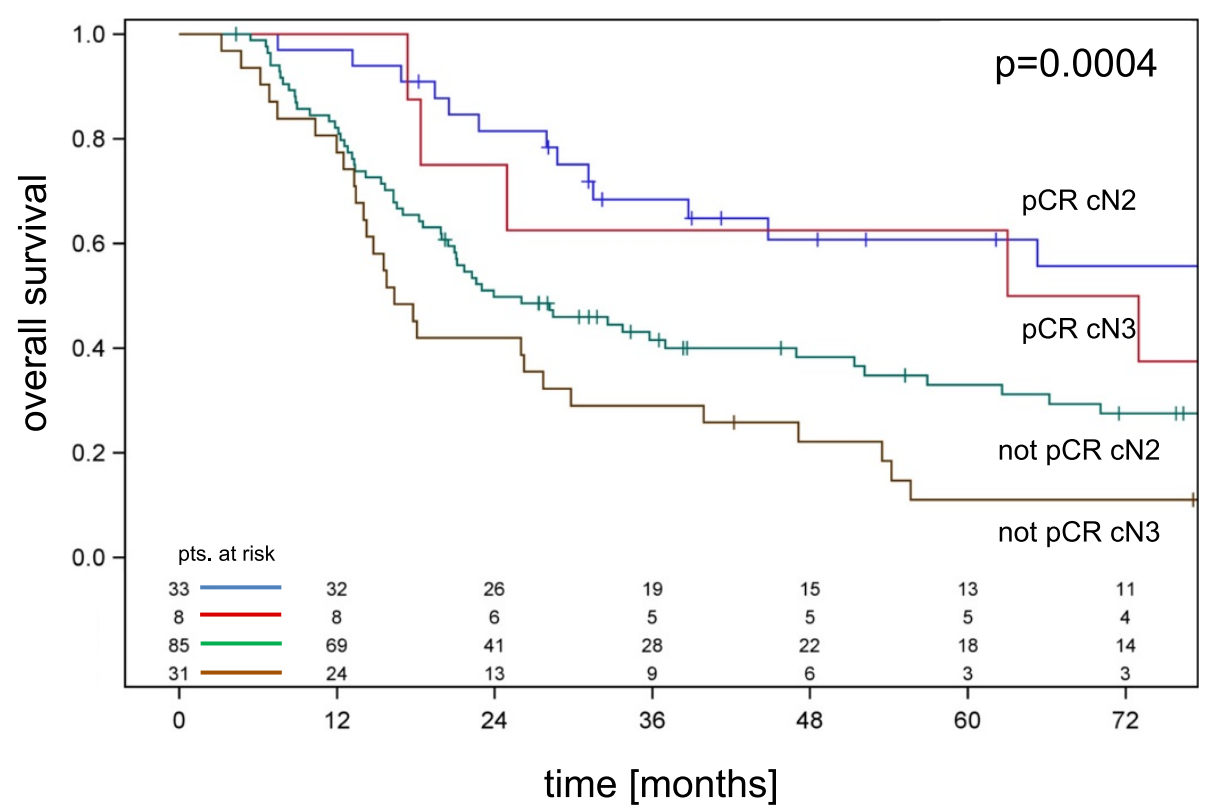

Figure 4 Kaplan Meier survival curves for the subgroups of patients with pathological complete remission (pCR), and without pCR (not-pCR) according to nodal category cNO-1, CN2, and cN3.

[9-11]. To substantiate the notion that $\mathrm{pCR}$ is associated with prolonged survival by improved long-term tumor control, the association between $\mathrm{pCR}$ and time to progression was analysed using PHA, and pCR was again observed as positive prognostic factor. The specificity for the prediction of long term survivors decreased substantially when the histopathologic criterium was broadened from $\mathrm{pCR}$ to $\mathrm{MNC}$ or regression grade $\geq 2 \mathrm{~B}$. Devitalisation of tumor in both the primary site and lymph nodes seems to be important to improve prognosis of patients treated with induction chemotherapy followed by radiochemotherapy in lung cancer. A similar observation was made in breast cancer after neoadjuvant chemotherapy. Disease-free survival was highest in breast cancer patients with $\mathrm{PCR}$ at the primary site and the involved lymph nodes [20]. The hazard ratio for disease-free survival in comparison to patients without histopathologic response increased from 0.45 for patients with no in situ residuals in the primary tumor and the lymph nodes to $0.52,0.62$, and 0.73 , respectively, when patients with in situ residuals at the primary site, or patients with residually involved nodes but no invasive breast cancer at the primary site, or patients with focal invasive disease were included in the histopathologic response definition [21]. It was therefore concluded that ypTis, ypT1mic and ypN+ residuals alone are associated with an increased relapse risk and should not be considered in the definition of positive histopathologic response.

Downstaging to $\mathrm{pCR}$ after neoadjuvant radiochemotherapy can improve the prognosis for $\mathrm{cN} 2$ and $\mathrm{cN} 3 \mathrm{pa}-$ tients and yields favorable long-term survival. In the cN2-3 group of our patients achieving pCR, five-year survival was $61 \%$, well within the range of other studies [11]. The observation that $\mathrm{pCR}$ alone is a predictor of better prognosis is reflected by the position of $\mathrm{pCR}$ in the top node of the regression tree.

Selection of the optimal candidates for surgery remains a crucial point in daily clinical practice and is strongly influenced by current restaging capabilities [11,22]. Even considering the whole spectrum of procedures (from noninvasive radiological tools to more invasive surgical approaches), the restaging assessment often fails to predict the actual pathological response in this situation. Volume response to neoadjuvant radiochemotherapy from sequential CT-studies has been found to be correlated with $\mathrm{PCR}$ and prognosis after neoadjuvant radiochemotherapy by this study and is an important preoperative prognostic factor that, however has not the predictive power of $\mathrm{pCR}$ itself. FDG-PET/CT has been investigated in this situation adding at least some valuable information on treatment response in these patient cohorts [23,24]. When using cutoff levels of deltaSUV $\mathrm{V}_{\max }$ around 0.3 to define responders a positive correlation with $\mathrm{pCR}$ was observed. Whether PET-response to neoadjuvant radiochemotherapy is a better prognostic factor for overall survival than CT-response remains an open question.

The present study is a retrospective investigation of a group of consecutive patients treated at a single institution. Although the physicians' team has remained constant through the long duration of patients inclusion some changes of the induction as well as the concurrent treatment have taken place. Thus, some intrinsic bias cannot be excluded entirely and when translating our findings into a 
general setting of preoperative treatment this should be taken into account.

pCR improved the survival risk discrimination between equally sized low risk and high risk groups using a clinicopathologic prognostic model according to cross-validated Kaplan-Meier curves. There is a considerable interest to refine prognostic models for lung cancer by gene expression signatures and whole genome sequencing [25,26]. Given the dominant prognostic significance of $\mathrm{pCR}$ after neoadjuvant radiochemotherapy using cisplatin containing doublets, pCR should be included as a prognostic factor in studies of patients after neoadjuvant radiochemotherapy. If further treatments are considered after neoadjuvant radiochemotherapy and resection, as in the Intergroup 0139 or the RTOG $02-29$ trial [2,6], the prognostic model including pCR might help to identify patients who benefit most from additional postoperative treatment or treatment intensification, respectively.

\section{Abbreviations}

CMH: Cochrane-Mantel-Haenszel; CR: Complete remission; CT: Computed tomography; CTC: Common toxicity criteria; MNC: Mediastinal nodal clearance; NC: No change; NOS: Not otherwise specified; NSCLC: Non-small cell lung cancer; OS: Overall survival; pCR: Pathologic complete remission; PCR-T: Pathologic complete remission in the primary tumor; PD: Progressive disease; PET/CT: Positron emission tomography/computed tomography; PHA: Proportional hazard analysis; PR: Partial remission; Reg-grade: Grade of remission; RPA: Recursive partitioning analysis; $S U V_{\text {max: }}$ Maximum standardized uptake value; TTP: Time to progression.

\section{Competing interests}

C. Pöttgen: honoraria from Roche. W. Eberhardt: honoraria from Astra Zeneca, Roche, Eli Lilly, Novartis, Pfizer, BayerSchering, Sanofiaventis, Boehringer Mannheim, BMS, GSK (advisory board member). T. Gauler: honoraria from BMS. M. Schuler: Research funding (to institution) from Boehringer Ingelheim, Novartis; honoraria (consulting, medical education) and travel support from AstraZeneca, Boehringer Ingelheim, Lilly, Novartis, Pfizer, Roche.

\section{Authors' contributions}

$\mathrm{CP}, \mathrm{MStu}, \mathrm{BG}$ participated in the design of the study and performed the statistical analysis. DT, TG, VJ, LF, JAJ, EG, JW, SW, MH, MS, GS, WE conceived of the study, and participated in its design and coordination, and helped to draft the manuscript. All authors have read and approved the final manuscript.

\section{Acknowledgments}

There has been no funding to this study.

\section{Author details \\ 'Department of Radiotherapy, West German Cancer Center, University of Duisburg-Essen, Essen, Germany. Institute of Pathology and Neuropathology, West German Cancer Center, University of Duisburg-Essen, Essen, Germany. ${ }^{3}$ Department of Medical Oncology, West German Cancer Center, University of Duisburg-Essen, Essen, Germany. ${ }^{4}$ Institute of Cell Biology (Cancer Research), University of Duisburg-Essen, Essen, Germany. ${ }^{5}$ Division of Interventional Pneumology, Ruhrlandklinik, West German Lung Center, University of Duisburg-Essen, Essen, Germany. ${ }^{6}$ Division of Thoracic Surgery and Thoracic Endoscopy, Ruhrlandklinik, West German Lung Center, University of Duisburg-Essen, Essen, Germany. ${ }^{7}$ Division of Thoracic Oncology, Ruhrlandklinik, West German Lung Center, University of Duisburg-Essen, Essen, Germany. ${ }^{8}$ German Cancer Consortium (DKTK), Heidelberg, Germany.}

Received: 10 March 2014 Accepted: 28 April 2015

Published online: 06 May 2015

\section{References}

1. Albain KS, Rusch WW, Crowley J, Rice TW, Turrisi 3rd AT, Weick JK, et al. Concurrent cisplatin/etoposide plus chest radiotherapy followed by surgery for stages IIIA (N2) and IIIB non-small-cell lung cancer: mature results of Southwest Oncology Group Phase II study 8805. J Clin Oncol. 1995;13:1880-92.

2. Albain KS, Swann RS, Rusch WW, Turrisi 3rd AT, Shepherd FA, Smith C, et al. Radiotherapy plus chemotherapy with or without surgical resection for stage III non-small-cell lung cancer: a phase III randomized controlled trial. Lancet. 2009:374:379-86.

3. Thomas M, Rübe C, Hoffknecht P, Macha HN, Freitag L, Linder A, et al. Effect of preoperative chemoradiation in addition to preoperative chemotherapy: a randomized trial in stage III non-small-cell lung cancer. Lancet Oncol. 2008;9:636-48

4. van Meerbeeck JP, Kramer GWPM, van Schill PEY, Legrand C, Smit EF, Schramel F, et al. Randomized controlled trial of resection versus radiotherapy after induction chemotherapy in stage IIIA-N2 non-small-cell lung cancer. J Natl Cancer Inst. 2007;99:442-50.

5. Kim AW, Liptay MJ, Bonomi P, Warren WH, Basu S, Farlow EC, et al. Neoadjuvant chemoradiation for clinically advanced non-small cell lung cancer: An analysis of 233 patients. Ann Thorac Surg. 2011;92:233-43.

6. Suntharalingam M, Paulus R, Edelman MJ, Krasna M, Burrows W, Gore E, et al. Radiation Oncology Group Protocol 02-29: A phase II trial of neoadjuvant therapy with concurrent chemotherapy and full-dose radiation therapy followed by surgical resection and consolidative therapy for locally advanced non-small cell carcinoma of the lung. Int J Radiat Oncol Biol Phys. 2012;84:456-63.

7. Pöttgen C, Eberhardt W, Graupner B, Theegarten D, Gauler T, Freitag L, et al. Accelerated hyperfractionated radiotherapy within trimodality therapy concepts for stage IIIA/B non-small cell lung cancer: markedly higher rate of pathologic complete remissions than with conventional fractionation. Eur J Cancer. 2013;49:2107-15.

8. Eberhardt W, Wilke H, Stamatis G, Stuschke M, Harstrick A, Menker H, et al. Preoperative chemotherapy followed by concurrent chemoradiation therapy based on hyperfractionated accelerated radiotherapy and definitive surgery in locally advanced non-small-cell lung cancer. Mature results of a phase II trial. J Clin Oncol. 1998;16:622-34.

9. Friedel G, Budach W, Dippon J, Spengler W, Eschmann SM, Pfannenberg C, et al. Phase II trial of a trimodality regimen for stage III non-small-cell lung cancer using chemotherapy as induction treatment with concurrent hyperfractionated chemoradiation with carboplatin and paclitaxel followed by subsequent resection: a single-center study. J Clin Oncol. 2010;28:942-8.

10. Cerfolio RJ, Bryant AS, Jones VL, Cerfolio RM. Pulmonary resection after concurrent chemotherapy and high dose (60 Gy) radiation for non-small cell lung cancer is safe and may provide increased survival. Eur J Cardiothorac Surg. 2009;35:718-23.

11. Lococo F, Cesario A, Margaritora S, Dall'Armi V, Mattei F, Romano R, et al. Long-term results in patients with pathological complete response after induction radiochemotherapy followed by surgery for locally advanced non-small-cell lung cancer. Eur J Cardiothorac Surg. 2013;43:e71-81.

12. Stamatis G, Djuric D, Eberhardt W, Pöttken C, Zaboura G, Fechner S, et al. Postoperative morbidity and mortality after induction chemoradiotherapy for locally advanced lung cancer: an analysis of 350 operated patients. Eur J Cardiothorac Surg. 2002;22:292-7.

13. Trotti A, Byhardt R, Stetz J, Gwede C, Corn B, Fu K, et al. Common toxicity criteria: version 2.0. an improved reference for grading the acute effects of cancer treatment: impact on radiotherapy. Int J Radiat Oncol Biol Phys. 2000;:47:13-47.

14. Junker K, Langner K, Klinke F, Bosse U, Thomas M. Grading of tumor regression in non-small cell lung cancer. Morphology and prognosis. Chest. 2001;120:1584-91.

15. Therasse P, Arbuck SG, Eisenhauer EA, Wanders J, Kaplan RS, Rubinstein L, et al. New guidelines to evaluate the response to treatment in solid tumors. J Natl Cancer Inst. 2000;92:205-16.

16. Harrell FE. The PHGLM procedure. SAS supplemental Library Series Guide, vol. Version 5. Cary, N.C.: SAS Institute; 1986.

17. Cox D: Regression models and lifetables. Journal of the Royal Statistical Society, Series B, 1972, 187-220.

18. Simon RM, Subramanian J, Li MC, Menezes S. Using cross-validation to evaluate predictive accuracy of survival risk classifiers based on high-dimensional data. Brief Bioinform. 2011;12:203-14. 
19. Segal MR. Regression Trees for Censored Data. Biometrics. 1988;44:35-47.

20. Prowell TM, Pazdur R. Pathological complete response and accelerated drug approval in early breast cancer. New Engl J Med. 2012;366:2438-41.

21. Von Minckwitz G, Untch M, Blohmer JU, Costa SD, Eidtmann H, Fasching PA, et al. Definition and impact of pathologic complete response on prognosis after neoadjuvant chemotherapy in various intrinsic breast cancer subtypes. J Clin Oncol. 2012;30:1796-804.

22. Nasir BS, Bryant AS, Minnich DJ, Wei B, Dransfield MT, Cerfolio RJ. The efficacy of restaging endobronchial ultrasound in patients with non-small cell lung cancer after preoperative therapy. Ann Thorac Surg. 2014;98:1008-12.

23. Choi NC, Fischman AJ, Niemierko A, Ryu JS, Lynch T, Wain J, et al. Doseresponse relationship between probability of pathologic tumor control and glucose metabolic rate measured with FDG PET after preoperative chemoradiotherapy in locally advanced non-small-cell lung cancer. Int J Radiat Oncol Biol Phys. 2002;54:1024-35.

24. Zhang C, Liu J, Tong J, Sun X, Song S, Huang G. 18 F-FDG-PET evaluation of pathological tumour response to neoadjuvant therapy in patients with NSCLC. Nucl Med Commun. 2013;34:71-7.

25. Zhu CQ, Ding K, Strumpf D, Weir BA, Meyerson M, Pennell N, et al, Prognostic and predictive gene signature for adjuvant chemotherapy in resected non-small-cell lung cancer. J Clin Oncol. 2010;28:4417-24.

26. Daniels M, Goh F, Wright CM, Sriram KB, Relan V, Clarke BE, et al. Whole genome sequencing for lung cancer. J Thorac Dis. 2012;4:155-63.

\section{Submit your next manuscript to BioMed Central and take full advantage of:}

- Convenient online submission

- Thorough peer review

- No space constraints or color figure charges

- Immediate publication on acceptance

- Inclusion in PubMed, CAS, Scopus and Google Scholar

- Research which is freely available for redistribution 